\title{
Electron impact excitation of Ar XVII ${ }^{\star}$
}

\author{
K. M. Aggarwal and F. P. Keenan
}

\begin{abstract}
Department of Physics and Astronomy, Queen's University, Belfast BT7 1NN, Northern Ireland, UK e-mail: K.Aggarwal@qub.ac.uk
\end{abstract}

Received 12 May 2005 / Accepted 31 May 2005

\begin{abstract}
Energies for the lowest 49 levels among the $1 \mathrm{~s}^{2}$ and $1 \mathrm{~s} n \ell(n=2-5)$ configurations of Ar XVII have been calculated using the GRASP code of Dyall et al. (1989, Comput. Phys. Comm., 55, 424). Additionally, radiative rates, oscillator strengths, and line strengths are calculated for all electric dipole (E1), magnetic dipole (M1), electric quadrupole (E2), and magnetic quadrupole (M2) transitions among these levels. Furthermore, collision strengths have also been calculated for all the 1176 transitions among the above 49 levels using the Dirac Atomic $R$-matrix Code (DARC) of Norrington \& Grant (2005, Comput. Phys. Commun., in preparation), over a wide energy range up to $580 \mathrm{Ryd}$. Resonances have been resolved in the threshold region, and effective collision strengths have been obtained over a wide temperature range up to $\log T_{\mathrm{e}}=7.2 \mathrm{~K}$. Comparisons are made with the limited results available in the literature, and the accuracy of the data is assessed. Our energy levels are estimated to be accurate to better than $0.1 \%$, whereas results for other parameters are probably accurate to better than $20 \%$.
\end{abstract}

Key words. atomic data - atomic processes

\section{Introduction}

In two recent papers, we (Aggarwal et al. 2005; McKeown et al. 2004) have reported calculations of energy levels and radiative rates for transitions in Ar XIII - Ar XVI, and in this work we report similar results for transitions in Ar XVII. Additionally, we also present our calculations for collision strengths $(\Omega)$ and effective collision strengths $(\Upsilon)$ for transitions among the lowest 49 levels of the $1 \mathrm{~s}^{2}, 1 \mathrm{~s} 2 \mathrm{~s}, 1 \mathrm{~s} 2 \mathrm{p}, 1 \mathrm{~s} 3 \ell, 1 \mathrm{~s} 4 \ell$ and $1 \mathrm{~s} 5 \ell$ configurations.

Emission lines from the spectra of highly ionized argon have been observed in the UV, EUV and X-ray spectra of solar, stellar and other astrophysical plasmas by many space missions, such as SOHO, Chandra and XMM Newton. Some of the observed lines from the spectra of Ar XIV - XVIII have been specifically listed by Dere et al. (2001), and a complete list of lines over a wide range of wavelengths for many ions of argon are available in the CHIANTI database at http://wwwsolar.nrl.navy.mil/chianti .html.Emission lines of He-like ions, including those from Ar XVII, are also a prominent feature of the X-ray spectra of tokamak plasmas (Keenan et al. 1987; Phillips et al. 1994), which arise from impurity elements.

An analysis of observed spectra provides information on the temperature, density and chemical composition of the plasma. However, such an analysis requires information for a

* Tables 2-6 are available only in electronic form at the CDS via anonymous ftp to cdsarc.u-strasbg. fr (130.79.128.5) or via http://cdsweb.u-strasbg.fr/cgi-bin/qcat? J/A+A/441/831 wide range of atomic parameters, including energy levels, radiative rates, and excitation rate coefficients. Since there is a paucity of measurements for these atomic parameters, theoretical results are of vital importance. A few calculations have been performed in the past by some authors, but most of these are limited to a few energy levels and/or transitions. Therefore, in this work we attempt to report results for a wider range of energy levels, and hence for a larger number of transitions. Furthermore, most of the available theoretical data are confined to the radiative rates for allowed and inter-combination (E1) transitions alone, whereas we here report similar data for other types of transitions as well, namely electric quadrupole (E2), magnetic dipole (M1) and magnetic quadrupole (M2), because these data are also required in the analysis and modelling of plasmas.

To report results for above named atomic parameters, we have employed the fully relativistic GRASP (General purpose Relativistic Atomic Structure Package) code of Dyall et al. (1989) for the generation of wavefunctions, and the Dirac Atomic $R$-matrix Code (DARC) of Norrington \& Grant (2005) for the computations of $\Omega$, and subsequently of $\Upsilon$.

\section{Energy levels}

The $1 \mathrm{~s}^{2}, 1 \mathrm{~s} 2 \mathrm{~s}, 1 \mathrm{~s} 2 \mathrm{p}, 1 \mathrm{~s} 3 \ell, 1 \mathrm{~s} 4 \ell$ and $1 \mathrm{~s} 5 \ell$ configurations of Ar XVII give rise to 49 fine-structure levels, listed in Table 1. Our calculated energies obtained from the GRASP code, with and without including the Breit and QED effects, are given in this table along with those from the CHIANTI database and the experimental compilations of NIST 
(http: //physics.nist.gov/PhysRefData). For our calculations, we have used the option of extended average level (EAL), in which a weighted (proportional to $2 j+1$ ) trace of the Hamiltonian matrix is minimized. This produces a compromise set of orbitals describing closely lying states with moderate accuracy. Our calculations are in the $j j$ coupling and configuration interaction (CI) has been included among the above stated 15 configurations. The inclusion of Breit and QED effects not only lowers the energies by a maximum of 0.3 Ryd (see, for example, levels 33-49), but also (slightly) alters the orderings in a few instances, such as for levels 21-24. However, we have retained the original orderings of the Coulomb energies, because these are the ones adopted in the subsequent tables.

It is clear from Table 1 that experimental energies are not available for all the levels, but the agreement with our results is within 0.2 Ryd (better than $0.1 \%$ ), which is highly satisfactory. The energy levels of the CHIANTI database are from the calculations of Sampson et al. (1983). These results are slightly higher than our values (last column) or those of NIST, but agree closely with our data obtained without the inclusion of the Breit and QED effects. Additionally, energies of Sampson et al. are non-degenerate for many levels, such as for levels 37-49. However, Sampson et al. have clearly stated that their calculations are primarily for generating large quantities of collision strengths, and are not for accurate determination of transition energies, i.e. energy levels. For the same reason, they have estimated the accuracy of their energy levels to be within $1 \%$, which is seen to be the case from Table 1. To conclude, we can state with confidence that our calculations have improved upon the energy levels of Sampson et al., which have also been beneficial in determining the level orderings.

\section{Radiative rates}

The absorption oscillator strength $\left(f_{i j}\right)$ and radiative rate $\mathrm{A}_{j i}$ (in $\mathrm{s}^{-1}$ ) for a transition $i \rightarrow j$ are related by the following expression:

$f_{i j}=\frac{m c}{8 \pi^{2} e^{2}} \lambda_{j i}{ }^{2} \frac{\omega_{j}}{\omega_{i}} A_{j i}=1.49 \times 10^{-16} \lambda_{j i}^{2}\left(\omega_{j} / \omega_{i}\right) A_{j i}$

where $m$ and $e$ are the electron mass and charge, respectively, $c$ is the velocity of light, $\lambda_{j i}$ is the transition energy/wavelength in $\AA$, and $\omega_{i}$ and $\omega_{j}$ are the statistical weights of the lower $i$ and upper $j$ levels, respectively. Similarly, the oscillator strength $f_{i j}$ (dimensionless) and the line strength $S$ (in atomic unit, $1 \mathrm{au}=6.460 \times 10^{-36} \mathrm{~cm}^{2} \mathrm{esu}^{2}$ ) are related by the following standard equations:

For the electric dipole (E1) transitions:

$A_{j i}=\frac{2.0261 \times 10^{18}}{\omega_{j} \lambda_{j i}^{3}} S^{\mathrm{E} 1} \quad$ and $\quad f_{i j}=\frac{303.75}{\lambda_{j i} \omega_{i}} S^{\mathrm{E} 1}$,

for the magnetic dipole (M1) transitions:

$A_{j i}=\frac{2.6974 \times 10^{13}}{\omega_{j} \lambda_{j i}^{3}} S^{\mathrm{M} 1} \quad$ and $\quad f_{i j}=\frac{4.044 \times 10^{-3}}{\lambda_{j i} \omega_{i}} S^{\mathrm{M} 1}$ for the electric quadrupole (E2) transitions:

$A_{j i}=\frac{1.1199 \times 10^{18}}{\omega_{j} \lambda_{j i}^{5}} S^{\mathrm{E} 2} \quad$ and $\quad f_{i j}=\frac{167.89}{\lambda_{j i}^{3} \omega_{i}} S^{\mathrm{E} 2}$,

and for the magnetic quadrupole (M2) transitions:

$A_{j i}=\frac{1.4910 \times 10^{13}}{\omega_{j} \lambda_{j i}^{5}} S^{\mathrm{M} 2} \quad$ and $\quad f_{i j}=\frac{2.236 \times 10^{-3}}{\lambda_{j i}^{3} \omega_{i}} S^{\mathrm{M} 2}$

In Table 2 we present transition energies $\left(\Delta E_{i j}\right.$ in $\AA$ ), radiative rates $\left(A_{j i}\right.$ in $\left.\mathrm{s}^{-1}\right)$, oscillator strengths $\left(f_{i j}\right.$, dimensionless), and line strengths ( $S$ in au), in length form only, for all 336 electric dipole (E1) and 391 electric quadrupole (E2) transitions among the 49 levels of Ar XVII. The indices used to represent the lower and upper levels of a transition have already been defined in Table 1. Similar results for 316 magnetic dipole (M1) and 410 magnetic quadrupole (M2) transitions are listed in Table 3. These results not only cover a wider range of transitions among larger number of levels than hitherto available in the literature, but are also for all possible transitions.

In Table 4 we compare our $f$-values for a limited set of transitions with those from the CHIANTI database, which are mainly from the calculations of Sampson et al. (1983). In this table we also list the ratio of our $f$-values in the length and velocity forms, in order to assess the accuracy of our results. For most of the transitions, the two forms of $f$-values agree within $20 \%$, which is quite satisfactory. However, for some transitions, such as 3-32, 4-32, 6-32 and 7-35, the two forms of $f$-values differ up to an order of magnitude, but their magnitudes are invariably small, i.e. $f \sim 10^{-3}$. Similarly, agreement with the CHIANTI $f$-values is within $20 \%$ for a majority of transitions, especially those with larger $f$-values. However, for some transitions, such as 3-27, 3-37 and 9-27, the two sets of $f$-values differ by an order of magnitude. These are comparatively stronger transitions, and $f_{L} / f_{V}$ is close to unity, which gives us confidence in our results. In general our results are higher with a few exceptions, such as for 6-13, 6-37 and 12-27 transitions, for which our $f$-values are lower by over an order of magnitude. However these transitions are comparatively weaker and $f_{L} / f_{V}$ is close to unity. Based on this comparison of our results with the corresponding data from CHIANTI, and between $f_{L}$ and $f_{V}$, we may state that for strong transitions ( $f \geq 0.01$ ) our radiative rates are accurate to $\sim 20 \%$, but scope remains for improvement for the weaker transitions.

\section{Collision strengths}

For the computations of collision strengths, we have employed the DARC program of Norrington \& Grant (2005). This program includes the relativistic effects in a systematic way, in both the target description and the scattering model. It is based on the $j j$ coupling scheme, and uses the Dirac-Coulomb Hamiltonian in the $R$-matrix approach. However, because of the inclusion of fine-structure in the definition of channel coupling, the matrix size of the Hamiltonian increases substantially. The $R$-matrix radius has been adopted to be $6.0 \mathrm{au}$, and 61 continuum orbitals have been included for each channel angular 
Table 1. Target levels of Ar XVII and their threshold energies (in Ryd).

\begin{tabular}{|c|c|c|c|c|c|c|}
\hline Index & Configuration & Level & Expt. $^{a}$ & 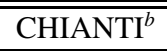 & $\overline{\text { GRASP }^{c}}$ & $\overline{\text { GRASP }^{d}}$ \\
\hline 1 & $1 \mathrm{~s}^{2}$ & ${ }^{1} \mathrm{~S}_{0}$ & 0.00000 & 0.000000 & 0.00000 & 0.00000 \\
\hline 2 & $1 \mathrm{~s} 2 \mathrm{~s}$ & ${ }^{3} S_{1}$ & 228.15015 & 228.225601 & 228.20039 & 227.97345 \\
\hline 3 & $1 s 2 p$ & ${ }^{3} \mathrm{P}_{0}^{\circ}$ & 229.52797 & 229.586395 & 229.55811 & 229.37311 \\
\hline 4 & $1 s 2 p$ & ${ }^{3} \mathrm{P}_{1}^{\circ}$ & 229.57456 & 229.683594 & 229.64185 & 229.41945 \\
\hline 5 & $1 \mathrm{~s} 2 \mathrm{~s}$ & ${ }^{1} \mathrm{~S}_{0}$ & 229.64803 & 229.813187 & 229.79482 & 229.59423 \\
\hline 6 & $1 s 2 p$ & ${ }^{3} \mathrm{P}_{2}{ }^{\circ}$ & 229.77757 & 229.942795 & 229.85488 & 229.61924 \\
\hline 7 & $1 s 2 p$ & ${ }^{1} \mathrm{P}_{1}^{\circ}$ & 230.75280 & 231.076797 & 230.91628 & 230.67177 \\
\hline 8 & $1 \mathrm{~s} 3 \mathrm{~s}$ & ${ }^{3} \mathrm{~S}_{1}$ & 270.03877 & 270.118805 & 270.10078 & 269.86520 \\
\hline 9 & $1 s 3 p$ & ${ }^{3} \mathrm{P}_{0}^{\circ}$ & 270.41842 & 270.507599 & 270.48924 & 270.26436 \\
\hline 10 & $1 s 3 p$ & ${ }^{3} \mathrm{P}_{1}^{\circ}$ & 270.43266 & 270.539978 & 270.51412 & 270.27814 \\
\hline 11 & $1 \mathrm{~s} 3 \mathrm{~s}$ & ${ }^{1} \mathrm{~S}_{0}$ & 270.43505 & 270.539978 & 270.56681 & 270.33115 \\
\hline 12 & $1 s 3 p$ & ${ }^{3} \mathrm{P}_{2}{ }^{\circ}$ & 270.49291 & 270.604797 & 270.57739 & 270.33735 \\
\hline 13 & $1 \mathrm{~s} 3 \mathrm{~d}$ & ${ }^{3} \mathrm{D}_{1}$ & 270.70342 & 270.831604 & 270.76091 & 270.52312 \\
\hline 14 & $1 \mathrm{~s} 3 \mathrm{~d}$ & ${ }^{3} \mathrm{D}_{2}$ & 270.70332 & 270.831604 & 270.76537 & 270.52336 \\
\hline 15 & $1 \mathrm{~s} 3 \mathrm{~d}$ & ${ }^{3} \mathrm{D}_{3}$ & 270.72730 & 270.864014 & 270.78817 & 270.54629 \\
\hline 16 & $1 \mathrm{~s} 3 \mathrm{~d}$ & ${ }^{1} \mathrm{D}_{2}$ & 270.73840 & 270.896393 & 270.79950 & 270.55973 \\
\hline 17 & $1 s 3 p$ & ${ }^{1} \mathrm{P}_{1}^{\circ}$ & 270.75700 & 270.896393 & 270.90193 & 270.65251 \\
\hline 18 & $1 \mathrm{~s} 4 \mathrm{~s}$ & ${ }^{3} \mathrm{~S}_{1}$ & 284.49700 & 284.569214 & 284.51374 & 284.33206 \\
\hline 19 & $1 s 4 p$ & ${ }^{3} \mathrm{P}_{0}{ }^{\circ}$ & 284.65550 & 284.731201 & 284.66948 & 284.51012 \\
\hline 20 & $1 s 4 p$ & ${ }^{3} \mathrm{P}_{1}^{\circ}$ & 284.66200 & 284.731201 & 284.67979 & 284.51602 \\
\hline 21 & $1 \mathrm{~s} 4 \mathrm{~s}$ & ${ }^{1} \mathrm{~S}_{0}$ & 284.66300 & 284.731201 & 284.70448 & 284.54750 \\
\hline 22 & $1 s 4 p$ & ${ }^{3} \mathrm{P}_{2}^{\circ}$ & 284.68700 & 284.763611 & 284.76417 & 284.54187 \\
\hline 23 & $1 \mathrm{~s} 4 \mathrm{f}$ & ${ }^{3} \mathrm{~F}_{2}{ }^{\circ}$ & & 284.893188 & 284.78987 & 284.59841 \\
\hline 24 & $1 \mathrm{~s} 4 \mathrm{f}$ & ${ }^{3} \mathrm{~F}_{3}{ }^{\circ}$ & & 284.893188 & 284.79216 & 284.59761 \\
\hline 25 & $1 \mathrm{~s} 4 \mathrm{f}$ & ${ }^{3} \mathrm{~F}_{4}^{\circ}$ & & 284.893188 & 284.79899 & 284.60335 \\
\hline 26 & $1 \mathrm{~s} 4 \mathrm{f}$ & ${ }^{1} \mathrm{~F}_{3}{ }^{\circ}$ & & 284.893188 & 284.80109 & 284.60404 \\
\hline 27 & $1 \mathrm{~s} 4 \mathrm{~d}$ & ${ }^{3} \mathrm{D}_{1}$ & 284.77650 & 284.860771 & 284.80115 & 284.61206 \\
\hline 28 & $1 \mathrm{~s} 4 \mathrm{~d}$ & ${ }^{3} \mathrm{D}_{2}$ & 284.77600 & 284.860771 & 284.80523 & 284.61259 \\
\hline 29 & $1 \mathrm{~s} 4 \mathrm{~d}$ & ${ }^{3} \mathrm{D}_{3}$ & 284.78659 & 284.893188 & 284.80532 & 284.62291 \\
\hline 30 & $1 \mathrm{~s} 4 \mathrm{~d}$ & ${ }^{1} \mathrm{D}_{2}$ & 284.79100 & 284.893188 & 284.80839 & 284.63107 \\
\hline 31 & $1 s 4 p$ & ${ }^{1} \mathrm{P}_{1}^{\circ}$ & 284.79900 & 284.893188 & 284.89237 & 284.69334 \\
\hline 32 & $1 \mathrm{~s} 5 \mathrm{~s}$ & ${ }^{3} S_{1}$ & 291.14600 & 291.211212 & 291.25271 & 290.94261 \\
\hline 33 & $1 s 5 p$ & ${ }^{3} \mathrm{P}_{0}^{\circ}$ & 291.22781 & 291.276001 & 291.30708 & 291.02186 \\
\hline 34 & $1 \mathrm{~s} 5 \mathrm{p}$ & ${ }^{3} \mathrm{P}_{1}^{\circ}$ & 291.23100 & 291.308411 & 291.32863 & 291.02504 \\
\hline 35 & $1 \mathrm{~s} 5 \mathrm{~s}$ & ${ }^{1} \mathrm{~S}_{0}$ & 291.23200 & 291.308411 & 291.32863 & 291.02873 \\
\hline 36 & $1 s 5 p$ & ${ }^{3} \mathrm{P}_{2}{ }^{\circ}$ & 291.24393 & 291.308411 & 291.33041 & 291.03802 \\
\hline 37 & $1 \mathrm{~s} 5 \mathrm{~d}$ & ${ }^{3} \mathrm{D}_{1}$ & & 291.373199 & 291.33041 & 291.08109 \\
\hline 38 & $1 \mathrm{~s} 5 \mathrm{~d}$ & ${ }^{3} \mathrm{D}_{2}$ & & 291.373199 & 291.35444 & 291.08138 \\
\hline 39 & $1 \mathrm{~s} 5 \mathrm{~d}$ & ${ }^{3} D_{3}$ & & 291.373199 & 291.35978 & 291.08625 \\
\hline 40 & $1 \mathrm{~s} 5 \mathrm{f}$ & ${ }^{3} \mathrm{~F}_{2}^{\circ}$ & & 291.373199 & 291.37420 & 291.08943 \\
\hline 41 & $1 \mathrm{~s} 5 \mathrm{f}$ & ${ }^{3} \mathrm{~F}_{3}{ }^{\circ}$ & & 291.373199 & 291.37423 & 291.08909 \\
\hline 42 & $1 \mathrm{~s} 5 \mathrm{~d}$ & ${ }^{1} \mathrm{D}_{2}$ & 291.29700 & 291.373199 & 291.37860 & 291.08971 \\
\hline 43 & $1 \mathrm{~s} 5 \mathrm{f}$ & ${ }^{3} \mathrm{~F}_{4}{ }^{\circ}$ & & 291.373199 & 291.37947 & 291.09206 \\
\hline 44 & $1 \mathrm{~s} 5 \mathrm{f}$ & ${ }^{1} \mathrm{~F}_{3}{ }^{\circ}$ & & 291.373199 & 291.37950 & 291.09237 \\
\hline 45 & $1 \mathrm{~s} 5 \mathrm{~g}$ & ${ }^{3} \mathrm{G}_{3}$ & & 291.373199 & 291.40201 & 291.09235 \\
\hline 46 & $1 \mathrm{~s} 5 \mathrm{~g}$ & ${ }^{3} \mathrm{G}_{4}$ & & 291.373199 & 291.40291 & 291.09214 \\
\hline 47 & $1 \mathrm{~s} 5 \mathrm{~g}$ & ${ }^{3} \mathrm{G}_{5}$ & & 291.373199 & 291.40919 & 291.09395 \\
\hline 48 & $1 \mathrm{~s} 5 \mathrm{~g}$ & ${ }^{1} \mathrm{G}_{4}$ & & 291.373199 & 291.41330 & 291.09409 \\
\hline 49 & $1 \mathrm{~s} 5 \mathrm{p}$ & ${ }^{1} \mathrm{P}_{1}^{\circ}$ & 291.30100 & 291.373199 & 291.41497 & 291.09523 \\
\hline
\end{tabular}

${ }^{a}$ NIST (http://physics.nist/gov/PhysRefData).

${ }^{b}$ Energy levels of Sampson et al. (1983) from CHIANTI database (http://wwwsolar.nrl .navy.mil/chianti.html).

${ }^{c}$ Present results from GRASP without Breit and QED effects.

${ }^{d}$ Present results from GRASP with Breit and QED effects. 


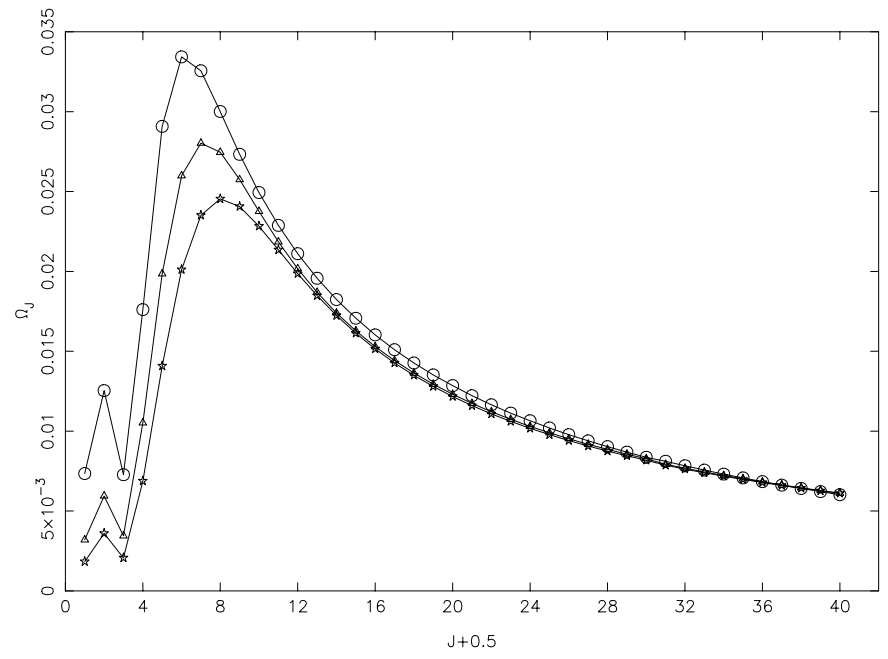

Fig. 1. Partial collision strengths for the $1 \mathrm{~s} 2 \mathrm{~s}^{3} \mathrm{~S}_{1}-1 \mathrm{~s} 2 \mathrm{p}^{3} \mathrm{P}_{1}^{\circ}(2-4)$ transition of Ar XVII, at three energies of 300 Ryd (circles), 400 Ryd (triangles), and 500 Ryd (stars).

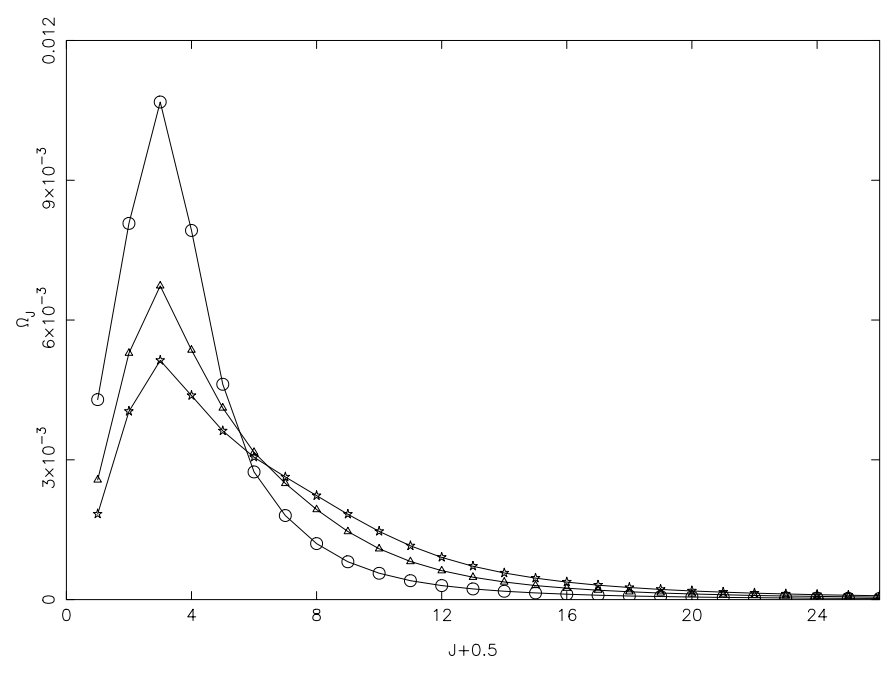

Fig. 2. Partial collision strengths for the $1 s 2 p^{3} P_{1}^{\circ}-1 s 2 p^{3} P_{2}^{\circ}(4-6)$ transition of Ar XVII, at three energies of 300 Ryd (circles), 400 Ryd (triangles), and 500 Ryd (stars).

momentum for the expansion of the wavefunction. This allows us to compute $\Omega$ up to an energy of 580 Ryd. The maximum number of channels for a partial wave is 217 , and the corresponding size of the Hamiltonian matrix is 13293 . In order to obtain convergence of $\Omega$ for all transitions and at all energies, we have included all partial waves with angular momentum $J \leq 39.5$, although a higher range would have been preferable for the convergence of allowed transitions, especially those with $\Delta n=0$. However, to account for the inclusion of higher neglected partial waves, we have included a top-up, based on the Coulomb-Bethe approximation for allowed transitions and geometric series for forbidden transitions.

In Figs. 1-3 we show the variation of $\Omega$ with angular momentum $J$ at three energies of 300, 400 and 500 Ryd, and for three transitions, namely $2-4\left(1 \mathrm{~s} 2 \mathrm{~s}{ }^{3} \mathrm{~S}_{1}-1 \mathrm{~s} 2 \mathrm{p}{ }^{3} \mathrm{P}_{1}^{\circ}\right)$, 4-6 (1s $\left.2 \mathrm{p}^{3} \mathrm{P}_{1}^{\circ}-1 \mathrm{~s} 2 \mathrm{p}{ }^{3} \mathrm{P}_{2}^{\circ}\right)$ and $4-14\left(1 \mathrm{~s} 2 \mathrm{p}^{3} \mathrm{P}_{1}^{\circ}-1 \mathrm{~s} 3 \mathrm{~d}^{3} \mathrm{D}_{2}\right)$, which are "elastic" (i.e. allowed with $\Delta n=0$ ), parity

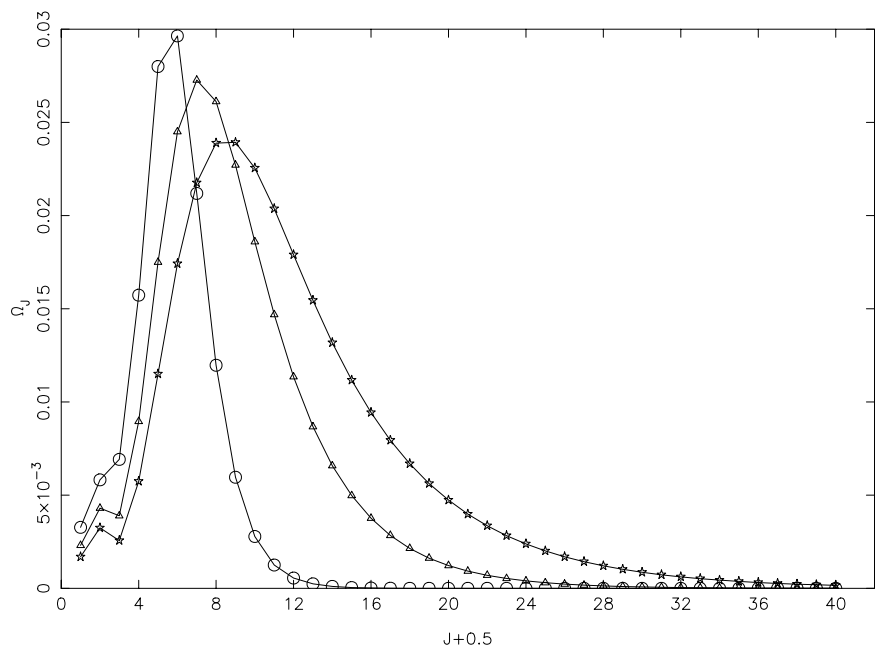

Fig. 3. Partial collision strengths for the $1 s 2 p{ }^{3} P_{1}^{\circ}-1 s 3 d{ }^{3} D_{2}$ (4-14) transition of Ar XVII, at three energies of 300 Ryd (circles), 400 Ryd (triangles), and 500 Ryd (stars).

forbidden, and allowed $(\Delta n \neq 0)$, respectively. For the forbidden and allowed transitions shown in Figs. 2 and 3, $\Omega$ have fully converged at all energies, including the highest energy of our calculations. However, for the "elastic" transitions our range of partial waves in not sufficient for the convergence of $\Omega$, as shown in Fig. 1. For such transitions a top-up from the Coulomb-Bethe approximation is quite significant.

In Table 5 we present our results of $\Omega$ for all transitions in a wider energy range ( $300 \leq E \leq 550 \mathrm{Ryd}$ ), but above thresholds. The indices adopted to represent a transition are already given in Table 1 . These results of $\Omega$ are not directly applicable in any diagnostic or modelling work, but are very useful in assessing the accuracy of a calculation, and will be helpful for future comparisons.

\section{Effective collision strengths}

Effective collision strengths $\Upsilon$ are obtained after integrating $\Omega$ over a Maxwellian distribution of electron velocities, i.e.

$\Upsilon\left(T_{\mathrm{e}}\right)=\int_{0}^{\infty} \Omega(E) \exp \left(-E_{j} / k T_{\mathrm{e}}\right) \mathrm{d}\left(E_{j} / k T_{\mathrm{e}}\right)$

where $E_{j}$ is the incident energy of the electron with respect to the final state of the transition, $k$ is Boltzmann's constant, and $T_{\mathrm{e}}$ is the electron temperature in $\mathrm{K}$. Once the value of $\Upsilon$ is known for a transition, the corresponding value of the excitation $q(i, j)$ and de-excitation $q(j, i)$ rate coefficients can be easily obtained from the following simple relations:

$q(i, j)=\frac{8.63 \times 10^{-6}}{\omega_{i} T_{\mathrm{e}}^{1 / 2}} \Upsilon \exp \left(-E_{i j} / k T_{\mathrm{e}}\right) \quad \mathrm{cm}^{3} \mathrm{~s}^{-1}$

and

$q(j, i)=\frac{8.63 \times 10^{-6}}{\omega_{j} T_{\mathrm{e}}^{1 / 2}} \Upsilon \quad \mathrm{cm}^{3} \mathrm{~s}^{-1}$,

where $\omega_{i}$ and $\omega_{j}$ are the statistical weights of the initial $(i)$ and final $(j)$ states, respectively, and $E_{i j}$ is the transition energy. 
Since the threshold energy region is dominated by numerous resonances, $\Omega$ have been computed at a large number of energies in order to delineate these resonances. We have performed our calculations of $\Omega$ at $\sim 13000$ energies in the threshold region. Close to thresholds ( $\sim 0.1$ Ryd above a threshold) the energy mesh is $0.001 \mathrm{Ryd}$, and away from thresholds is 0.002 Ryd. Thus care has been taken to include as many resonances as possible, and with as fine a resolution as is computationally feasible. However, the energy gap between the $n=2$ and 3 levels is very wide, i.e. $\sim 40$ Ryd - see Table 1 . Therefore, in this energy region the mesh has been gradually increased to 0.01 Ryd.

The values of $\Upsilon$ so computed are listed in Table 6 at a series of electron temperatures in the range $5.2 \leq \log T_{\mathrm{e}} \leq 7.2 \mathrm{~K}$, which is fully sufficient for the application of the data to solar, astrophysical and fusion plasmas. In earlier work, Zhang \& Sampson (1987) have reported results of $\Upsilon$ for transitions among the lowest seven levels listed in Table 1. In their calculations, they have adopted the Coulomb-Born-Exchange method and have also included the contribution of resonances in an approximate way - see Zhang \& Sampson for details and discussion. Therefore, in Table 7 we compare our results of $\Upsilon$ with theirs at two common temperatures of $2.33 \times 10^{6}$ and $1.17 \times 10^{7} \mathrm{~K}$. Generally, the agreement between the two sets of results is within $20 \%$, which is highly satisfactory. However, for the $1-2\left(1 \mathrm{~s}^{2}{ }^{1} \mathrm{~S}_{0}-1 \mathrm{~s} 2 \mathrm{~s}{ }^{3} \mathrm{~S}_{1}\right)$ transition, our values of $\Upsilon$ are higher by up to $50 \%$, particularly towards the lower end of the temperature range. For this transition, we show our resonances in Fig. 4a in the entire threshold range. It is clear from this figure that there are dense and high resonances very close to the threshold, which have resulted in significantly higher values of $\Upsilon$ at lower temperatures. In Fig. $4 \mathrm{~b}$ we highlight these resonances in a narrow energy range below 231 Ryd. Since our calculations are in $j j$ coupling (i.e. state levels are degenerate) in comparison to the intermediate coupling adopted by Zhang \& Sampson, resonances are larger in our work. As a result of this, our values of $\Upsilon$ are generally higher, although there are some exceptions, such as for the 4-5 transition.

The other results of $\Upsilon$ available in the literature are by Keenan et al. (1987), who have interpolated results for transitions in Ar XVII from calculations for other He-like ions with nuclear charge $8 \leq Z \leq 26$. To be precise, they have adopted the $R$-matrix results of Kingston \& Tayal (1984) and Tayal \& Kingston (1984a,b, 1985) for C V, O VII and Mg XI, and the distorted-wave results of Pradhan (1985) for Ca XIX and Fe XXV. In Table 8 we compare our calculated results with their interpolated ones. For the lowest six transitions, the agreement between the two sets of $\Upsilon$ values is remarkably good, and within $10 \%$. However, for the remaining six transitions the discrepancy is up to a factor of two, and the present results are invariably higher. This is mainly because of our calculations being in the $j j$ coupling scheme, which gives rise to more accurate determination of resonances, especially at energies close to thresholds. As an example, we demonstrate our resonances for the $1 \mathrm{~s}^{2}{ }^{1} \mathrm{~S}_{0}-1 \mathrm{~s} 3 \mathrm{~s}{ }^{3} \mathrm{~S}_{1}$ (1-8) transition in Fig. 5, which clearly shows a few dense resonances close to the threshold. Considering that the earlier calculations, from which Keenan et al. interpolated their results, were in $L S$ coupling, involved

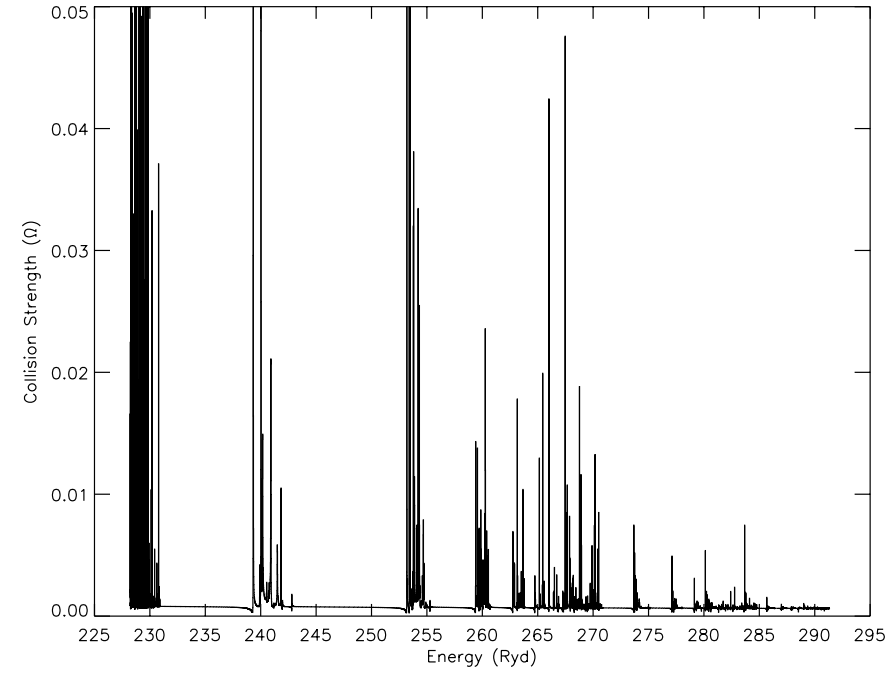

(a)

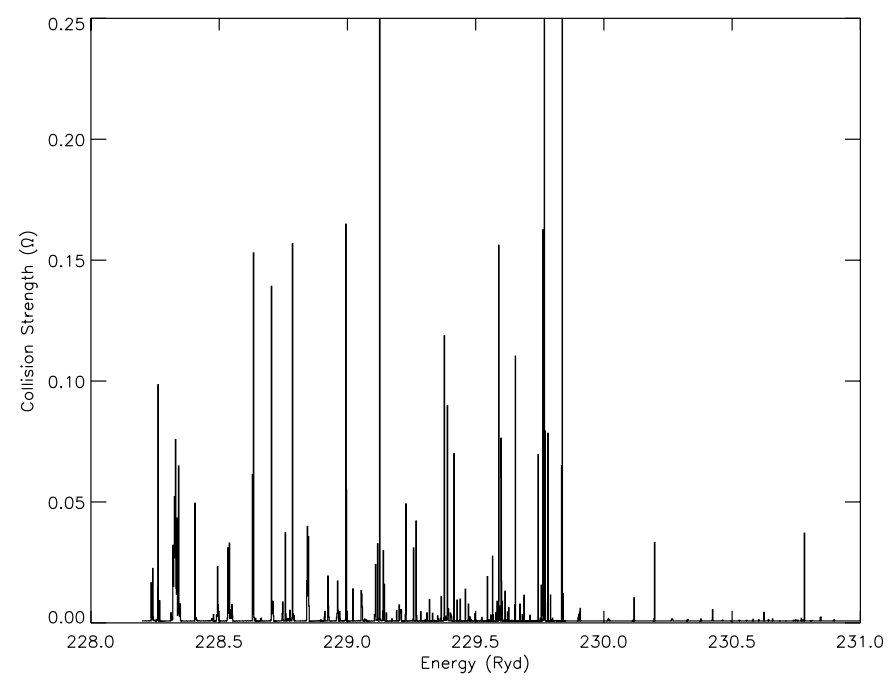

(b)

Fig. 4. Collision strengths for the $1 \mathrm{~s}^{2}{ }^{1} \mathrm{~S}_{0}-1 \mathrm{~s} 2 \mathrm{~s}{ }^{3} \mathrm{~S}_{1}$ (1-2) transition of Ar XVII.

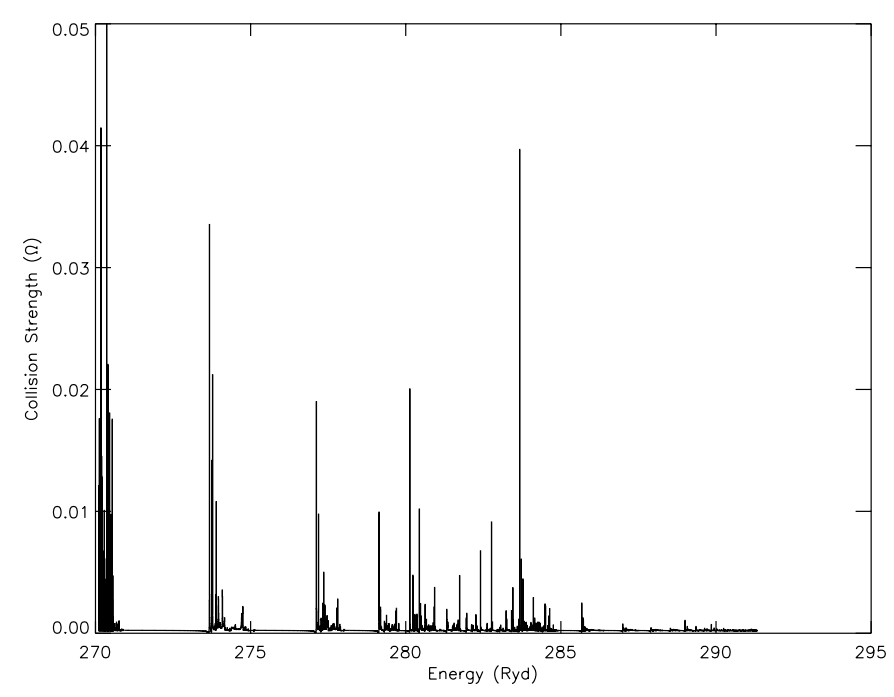

Fig. 5. Collision strengths for the $1 \mathrm{~s}^{2}{ }^{1} \mathrm{~S}_{0}-1 \mathrm{~s} 3 \mathrm{~s}{ }^{3} \mathrm{~S}_{1}$ (1-8) transition of Ar XVII. 
Table 7. Comparison of effective collision strengths ( $\Upsilon$ ) for transitions among the lowest 7 levels of Ar XVII. $\left(a \pm b \equiv a \times 10^{ \pm b}\right)$. First row: Zhang \& Sampson (1987), Second row: present results.

\begin{tabular}{lrllllll}
\hline \hline \multicolumn{2}{l}{ Transition } & \multicolumn{2}{l}{ Temperature (K) } & \multicolumn{2}{l}{ Transition } & \multicolumn{2}{c}{ Temperature (K) } \\
\hline$i$ & $j$ & $2.33+6$ & $1.17+7$ & $i$ & $j$ & $2.33+6$ & $1.17+7$ \\
\hline 1 & 2 & $9.78-4$ & $7.23-4$ & 3 & 4 & $2.49-2$ & $1.55-2$ \\
1 & 2 & $1.48-3$ & $8.98-4$ & 3 & 4 & $2.69-2$ & $1.72-2$ \\
1 & 3 & $5.07-4$ & $3.73-4$ & 3 & 5 & $2.10-3$ & $1.17-3$ \\
1 & 3 & $5.59-4$ & $4.14-4$ & 3 & 5 & $2.60-3$ & $1.48-3$ \\
1 & 4 & $1.57-3$ & $1.20-3$ & 3 & 6 & $2.63-2$ & $1.99-2$ \\
1 & 4 & $1.79-3$ & $1.35-3$ & 3 & 6 & $2.79-2$ & $2.18-2$ \\
1 & 5 & $1.64-3$ & $1.75-3$ & 3 & 7 & $1.34-2$ & $7.15-3$ \\
1 & 5 & $1.83-3$ & $1.89-3$ & 3 & 7 & $1.44-2$ & $8.21-3$ \\
1 & 6 & $2.53-3$ & $1.86-3$ & 4 & 5 & $1.89-2$ & $2.14-2$ \\
1 & 6 & $2.70-3$ & $2.06-3$ & 4 & 5 & $1.48-2$ & $1.78-2$ \\
1 & 7 & $5.31-3$ & $7.15-3$ & 4 & 6 & $9.17-2$ & $6.40-2$ \\
1 & 7 & $5.34-3$ & $7.25-3$ & 4 & 6 & $9.88-2$ & $7.14-2$ \\
2 & 3 & $2.09-1$ & $2.67-1$ & 4 & 7 & $3.88-2$ & $2.16-2$ \\
2 & 3 & $1.88-1$ & $3.04-1$ & 4 & 7 & $4.24-2$ & $2.52-2$ \\
2 & 4 & $6.09-1$ & $7.78-1$ & 5 & 6 & $1.06-2$ & $5.86-3$ \\
2 & 4 & $5.54-1$ & $8.90-1$ & 5 & 6 & $1.35-2$ & $7.56-3$ \\
2 & 5 & $9.22-3$ & $5.80-3$ & 5 & 7 & $6.17-1$ & $7.98-1$ \\
2 & 5 & $9.96-3$ & $6.24-3$ & 5 & 7 & $5.72-1$ & $9.12-1$ \\
2 & 6 & $9.96-1$ & $1.26-0$ & 6 & 7 & $6.15-2$ & $3.46-2$ \\
2 & 6 & $9.21-1$ & $1.49-0$ & 6 & 7 & $6.82-2$ & $4.12-2$ \\
2 & 7 & $2.72-2$ & $1.99-2$ & & & & \\
2 & 7 & $2.83-2$ & $2.36-2$ & & & & \\
\hline
\end{tabular}

Table 8. Comparison of effective collision strengths ( $\Upsilon$ ) for some transitions of Ar XVII. $\left(a \pm b \equiv a \times 10^{ \pm b}\right)$.

\begin{tabular}{lrllllll}
\hline \hline & \multicolumn{4}{c}{ Present Results } & \multicolumn{3}{c}{ Keenan et al. (1987) } \\
\hline \multicolumn{3}{l}{ Transition } & \multicolumn{5}{c}{ Temperature (log, K) } \\
\hline$i$ & $j$ & 6.80 & 7.00 & 7.20 & 6.80 & 7.00 & 7.20 \\
\hline 1 & 2 & $1.118-3$ & $9.531-4$ & $7.946-4$ & $9.454-4$ & $8.319-4$ & $7.184-4$ \\
1 & 3 & $4.873-4$ & $4.345-4$ & $3.729-4$ & $4.385-4$ & $3.931-4$ & $3.444-4$ \\
1 & 4 & $1.568-3$ & $1.408-3$ & $1.224-3$ & $1.356-3$ & $1.246-3$ & $1.131-3$ \\
1 & 5 & $1.847-3$ & $1.876-3$ & $1.886-3$ & $1.641-3$ & $1.673-3$ & $1.724-3$ \\
1 & 6 & $2.417-3$ & $2.157-3$ & $1.847-3$ & $2.191-3$ & $1.963-3$ & $1.719-3$ \\
1 & 7 & $6.271-3$ & $6.979-3$ & $7.698-3$ & $6.053-3$ & $6.694-3$ & $7.655-3$ \\
1 & 8 & $2.717-4$ & $2.352-4$ & $1.995-4$ & $1.428-4$ & $1.385-4$ & $1.307-4$ \\
1 & 9 & $1.306-4$ & $1.174-4$ & $1.016-4$ & $7.931-5$ & $7.679-5$ & $7.179-5$ \\
1 & 10 & $4.002-4$ & $3.625-4$ & $3.173-4$ & $2.527-4$ & $2.484-4$ & $2.379-4$ \\
1 & 11 & $3.803-4$ & $3.854-4$ & $3.841-4$ & $2.193-4$ & $2.462-4$ & $2.732-4$ \\
1 & 12 & $6.360-4$ & $5.715-4$ & $4.945-4$ & $2.917-4$ & $3.081-4$ & $3.068-4$ \\
1 & 13 & $6.430-5$ & $5.353-5$ & $4.327-5$ & & & \\
1 & 14 & $1.086-4$ & $9.390-5$ & $8.029-5$ & & & \\
1 & 15 & $1.435-4$ & $1.198-4$ & $9.700-5$ & & & \\
1 & 16 & $1.173-4$ & $1.119-4$ & $1.086-4$ & & & \\
1 & 17 & $1.270-3$ & $1.404-3$ & $1.518-3$ & $9.962-4$ & $1.143-3$ & $1.345-3$ \\
\hline
\end{tabular}


limited CI and energy levels (and hence less resonances), and economised on the number of partial waves included, the agreement between the two sets of results in Table 8 is perhaps more than satisfactory, but is more qualitative than quantitative.

\section{Conclusions}

In the present work, results for energy levels, radiative rates, collision strengths, and effective collision strengths for transitions among the lowest 49 levels of Ar XVII have been presented for all transitions. Additionally, results for radiative rates have been presented for four types of transitions, namely E1, E2, M1 and M2. The present set of results cover a wider range of transitions than hitherto available in the literature, and hence are likely to be useful for modelling and diagnostics of a variety of plasmas. Additionally, our calculations have been performed in the $j j$ coupling scheme, $\mathrm{CI}$ and relativistic effects have been included while generating wavefunctions, and a large range of partial waves has been adopted in order to achieve convergence in $\Omega$ values for a majority of transitions. Furthermore, resonances have been resolved in a fine energy mesh in order to improve upon the accuracy of the determined values of $\Upsilon$. Similarly, $\Omega$ have been computed in a wider energy range up to 580 Ryd in order to determine values of $\Upsilon$ up to a temperature of $\sim 1.6 \times 10^{7} \mathrm{~K}$. Based on comparisons made with the earlier available data for several atomic parameters, our energy levels are assessed to be accurate to $\sim 0.1 \%$, whereas the accuracy of other parameters is probably $\sim 20 \%$. As stated above, an overall improvement has been made, both in range and accuracy, over the existing results for a limited number of transitions. However, scope remains for further improvement mainly by extending the work to higher levels of the $n \geq 6$ configurations. Not only similar results for transitions with these higher levels will be useful for modelling work, but the accuracy of the present reported results will also be enhanced due to the inclusion of larger CI and hence more resonances arising from those. Until these improvements can be made in future work, we hope the present results will be highly useful for plasma applications.

Acknowledgements. This work has been financed by the Engineering and Physical Sciences and Particle Physics and Astronomy Research Councils of the UK. We would like to thank Dr. Patrick Norrington for providing his DARC code prior to publication, and F.P.K. is grateful to A.W.E. Aldermaston for the award of a William Penny Fellowship.

\section{References}

Aggarwal, K. M., Keenan, F. P., \& Nakazaki, S. 2005, A\&A, 436, 1141

Dere, K. P., Landi, E., Young, P. R., \& Del Zanna, G. 2001, ApJS, 134, 331

Dyall, K. G., Grant, I. P., Johnson, C. T., Parpia, F. A., \& Plummer, E. P. 1989, Comput. Phys. Commun., 55, 424

Keenan, F. P., McCann, S. M., \& Kingston, A. E. 1987, Phys. Scr., 35, 432

Kingston, A. E., \& Tayal, S. S. 1984, J. Phys. B, 16, 3465

McKeown, K., Aggarwal, K. M., Keenan, F. P., \& Rose, S. J. 2004, Phys. Scr., 70, 295

Norrington, P. H., \& Grant, I. P. 2005, Comput. Phys. Commun., in preparation

Phillips, K. J. H., Keenan, F. P., Harra, L. K., et al. 1994, J. Phys. B, 27,1939

Pradhan, A. K. 1985, ApJS, 59, 183

Sampson, D. H., Goett, S. J., \& Clark, R. E. H. 1983, At. Data Nucl. Data Tables, 29, 467

Tayal, S. S., \& Kingston, A. E. 1984a, J. Phys. B, 17, L145

Tayal, S. S., \& Kingston, A. E. 1984b, J. Phys. B, 17, 1383

Tayal, S. S., \& Kingston, A. E. 1985, J. Phys. B, 18, 2983

Zhang, H. L., \& Sampson, D. H. 1987, ApJS, 63, 487 